High Order Continuous Polygonal Patches

J.A. Gregory, J.M. Hahn and V.K.H. Lau 
BRUNEL UNIVERSITY

SEP 1919

LIBRARY

w9198943 


\title{
High Order Continuous Polygonal Patches
}

\author{
John A. Gregory \\ Vincent K.H. Lau \\ Department of Mathematics and Statistics, Brunel University, \\ Uxbridge, Middlesex UB8 3PH, England. \\ Jörg M. Hahn \\ Mercedes-Benz AG, Abt. DE/MK, \\ D-7032 Sindelfingen, Germany.
}

\begin{abstract}
A polygonal patch method is described which can be used to fill a polygonal hole within a given $\mathrm{k}^{\prime}$ th order continuous rectangular patch complex. The method is relatively easy to implement, since it only requires $C^{k}$ extensions of the rectangular patch complex defined in terms of the rectangular patch parameterizations. The method is illustrated by reference to $\mathrm{C}^{2}$ bicubic B-spline surfaces.
\end{abstract}

\section{Keywords}

Polygonal patches

\section{Introduction}

The parametric representation of surfaces in $C A G D$ is usually based on an assembly of patches with rectangular domains of definition. However, arbitrary surface topologies cannot be described by a regular rectangular patch framework. Either an arbitrary number of rectangular patches meeting at a vertex has to be allowed, or a polygonal patch has to be filled in. Here we consider the latter approach of constructing a polygonal patch. An $n$-sided patch will be exhibited which can be used to fill in a hole within a given $\mathrm{C}^{\mathrm{k}}$ rectangular patch complex, for any order of $k$. In particular, the case $k=2$ (curvature continuity) should be of practical interest in CAGD, for example, when filling a polygonal hole within a bicubic B-spline patch complex. 


\section{Polygonal Patches}

There have been several attempts to construct polygonal patches, see [Gregory, Lau and Zhou'90, Varady'87], but these only achieve $\mathrm{C}^{1}$ continuous joins with their rectangular patch neighbours. For example, [Charrot and Gregory'84] describe a pentagonal patch defined by a convex combination of parametric surfaces. As pointed out in [Gregory and Hahn'87b], this method cannot be immediately generalized to higher order continous surfaces, although a particular $\mathrm{C}^{2}$ solution is given in [Gregory and Hahn'89] The problem is that the continuity considerations cannot be treated within the given parameterizations, since the patches cannot be considered as being defined in a common parameter plane. The appropriate framework in which to examine continuity is that of $k$ 'th order 'geometric continuity' between the patches, see [Hahn'89], that is ${ }^{k}$ continuity under a reparameterization.

A general $k^{\prime}$ th order solution for the polygonal hole problem is given in an internal report [Gregory and Hahn'87a]. In that report, the rectangular patch data is reparameterized as $\mathrm{C}^{k}$ data around the exterior of a polygonal domain. This data is then extended into the interior of the polygon by a blending function interpolation method. Here, however, we adopt an approach which is much easier to implement. The rectangular patch complex is extended about each, corner of the hole in terms of the rectangular patch parameterizations. These extensions are then reparameterized onto the polygonal domain and blended to give the final polygonal patch. Theoretically, these two approaches to the problem are equivalent but practically there is a significant difference in the ease of implementation which leads us to recommend the method proposed here.

The construction of the reparameterizations must be considered with some care and most of the theoretical content of the paper is concerned with this problem. However, given the reparameterization functions (diffeomorphisms) proposed here, together with the $\mathrm{C}^{\mathrm{k}}$ extensions of the rectangular patch complex, it is then a simple matter to implement the polygonal patch method.

The polygonal hole problem is described in Section 2 and is followed, in Section 3, by a description of co-ordinate systems (co-ordinate charts) which are defined on the polygonal domain by central projections. These coordinate charts then form the basis of the reparameterization method used in the construction of the polygonal patch method in Section 4. This section contains most of the theory of the paper. In particular, the conditions to be satisfied by the reparameterization functions are developed. An alternative construction for the special case of a triangular hole is then considered in Section 5. In the final Section 6, we consider implementation of the polygonal patch schemes for the specific case of bicubic B-spline surfaces which contain polygonal holes. 


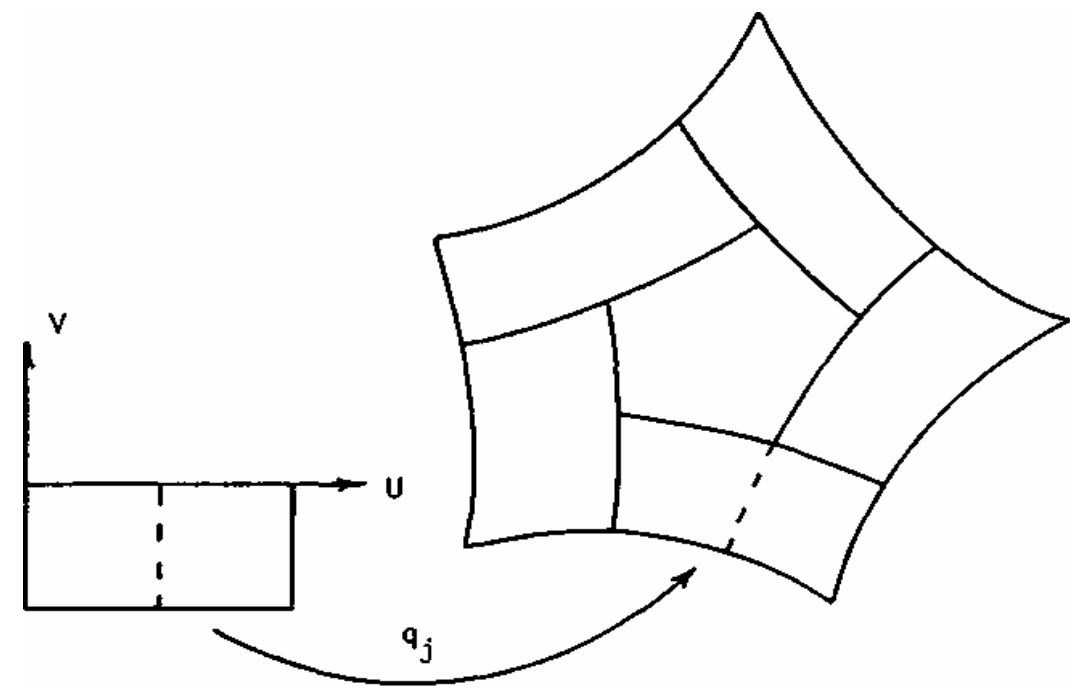

Figure 1: Five patches surrounding a polygonal hole

\section{The polygonal hole problem}

Assume that $\mathrm{q}_{\mathrm{i}} \mathrm{j}=0, \ldots, n-1$, describes a given $C^{k}$ parametric rectangular patch complex around an $n$-sided hole in $I R^{3}$, where $n \geq 3$. To make the exposition more concrete, suppose that

$$
q_{j}: \delta \rightarrow I R^{3}, \quad \delta=[0,2] \times[-1,0],
$$

where the segment $(\mathrm{s}, 0), 0 \leq \mathrm{s} \leq 1$, is mapped to the $j^{\prime}$ th boundary segment of the hole $I R^{3}$, see Figure 1. (In practice, $\mathrm{q}_{\mathrm{j}}$ will usually be composed of a sub-complex of two, or more, rectangular patches but it is mathematically convenient to represent these as one composite surface patch here.)

The patches are assumed to form a $C^{k}$ parametric patch complex in the sense that, for two adjacent patches $\mathrm{q}_{\mathrm{j}-1}$ and $\mathrm{q}_{\mathrm{j}}$, the composite map

$$
\mathrm{q}_{j-1, j}(u, v):= \begin{cases}\mathrm{q}_{j-l}(\sigma(Z u, v)), & (u, v) \in \sigma^{-1}(\delta)=[-1,0] \times[-1,1] \\ \mathrm{q}_{j}(u, v), & (u, v) \in \delta=[0,2] \times[-1,0],\end{cases}
$$

is $C^{k}$ continuous on the L-shaped domain $\sigma^{-1}(\delta) \cup \delta$, (6) $\mathrm{U}<5$, where

$$
\sigma(u, v):=(1-v, u), \quad \sigma^{-1}(u, v):=(v, 1-u),
$$

see Figure 2. In addition, the composite map will usually be $C^{k, k}$ continuous, that is, the partial derivatives

$$
\partial_{\ell, m} \mathrm{q}_{j-1-j}:=\frac{\partial^{\ell+m} \mathrm{q}_{j-1, j}}{\partial u^{\ell} \partial v^{m}}
$$




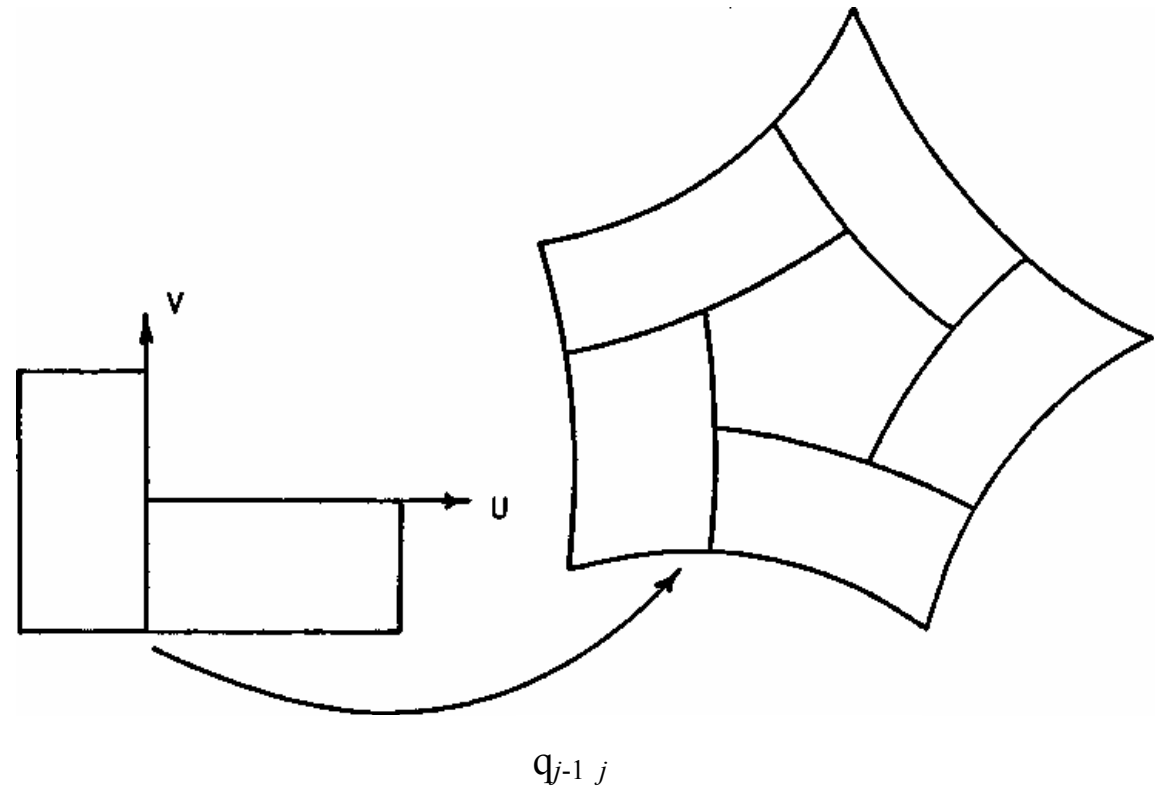

Figure 2: The composite map $\mathrm{q}_{j-1, j}$

exist for $0 \leq \ell, m, \leq k$, are continuous, and are independent of the order of differentiation.

Our objective is to extend the rectangular patch complex into the $n$ - sided hole in $I R^{3}$, with a surface patch $\mathrm{p}$ defined on a regular polygon. In order to achieve this goal, we assume that a $C^{k}$ extension of $\mathrm{q}_{j-1, j}$ into the positive quadrant is supplied for each $j=0, \ldots, n-1$. In particular, we assume that each $\mathrm{q}_{j-1, j}(u, v)$ is defined for $(u, v) \in[0,1]^{2}$. These $C^{k}$ extensions will then be defined on the regular polygon, through appropriate reparameterizations, and be blended together to form the polygonal patch $\mathrm{p}$.

The $C^{k}$ extensions of $\mathrm{q}_{j-1, j}$ into the unit square $[0,1]^{2}$ can easily be constructed in practice. For example, if the surrounding patch complex has a $C^{2,2}$ bi-cubic B-spline representation, then it is natural to extend $\mathrm{q}_{j-1, j}$ as a bi-cubic B-spline surface with appropriate additional control points. The important point to note is that the extensions are to be constructed with respect to a rectangular patch parameterization. The theory of the reparameterization of the $\mathrm{q}_{j-1, j}$ with respect to a regular polygonal domain is now considered in the following two sections.

\section{The polygonal domain}

Let $\Omega$ be a closed, regular, $n$-sided polygon in $I R^{2}$ with centre $0=(0,0)$ and sides of unit length. Its vertices are denoted by $X_{j}, j=0, \ldots, n-1$, and its edges are $E_{j}$, parameterized as

$$
E_{J}(s):=(1-s) X_{j}+s X_{j+1}
$$




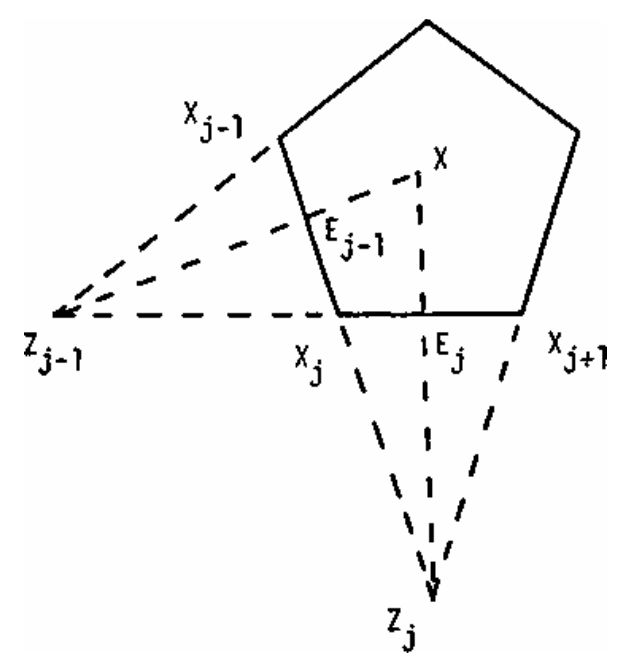

Figure 3: Central projection co-ordinate chart construction

In order to reparameterize the extensions $q_{i-1 . i}(u, v),(u, v) \in[0,1]^{2}$ on this regular polygon, we find it necessary to introduce co-ordinate charts $\phi_{i-1}, j=0, \ldots, n-1$, defined on $\Omega$. These co-ordinate charts respectively transform the angles at the vertices $X_{j}, j=0, \ldots, n-1$, to $\pi / 2$, and are defined here by central projections.

\section{The central projection co-ordinate chart}

Let $Z_{j}$ be the point of intersection of the edge $E_{j-1}$ with $E_{j+1}$ and, for a point $X \in \Omega$, let $u_{j}=u_{j}(X)$ be such that

$$
E_{J}\left(u_{j}\right):=\left(1-u_{j}\right) X_{j}+u_{j} X_{j+1}
$$

is the point of intersection of the edge $E_{j}$ with the ray from $Z_{j}$ through $\mathrm{X}$, see Figure 3. Let

$$
v_{j}:=1-u_{j-1,}
$$

Then

$$
E_{j-1}\left(1-v_{j}\right)=\left(1-v_{j}\right) X_{j}+v_{j} X_{j-1} .
$$

The co-ordinate chart $\phi_{j-1, j}$ is now defined by

$$
\phi_{j-1, j}(X):=\left(u_{j}(X), v_{j}(X)\right) .
$$

This chart maps $\Omega$ into $[0,1]^{2}$, where $X_{j}$ is mapped to $(0,0)$ and the two edges $E_{j}(u), E_{j-1}(1-v)$ are mapped onto $(u, 0),(0, v)$ respectively. Hence, the interior angle of the polygon at $X_{j}$ is mapped to $\pi / 2$.

The co-ordinate chart is conveniently computed as

$$
\phi_{j-1, j}:=\left(u_{j}, v_{j}\right):=\left(\frac{d_{j-1}}{d_{j-1}+d_{j+1}}, \frac{d_{j}}{d_{j-2}+d_{j}}\right),
$$


where $d_{j}=d_{j}(X)$ is the perpendicular distance of $X \in \Omega$ from the side $E_{j}$. In particular, in the case $n \geq 5$

$$
d_{j}=d_{j}(X):=\left\langle X_{j}-X, Z_{j}-0\right\rangle, /\left\|Z_{j}-0\right\|,
$$

where $<\bullet, \bullet>$ denotes the Euclidean scalar product in $I R^{2}$.

In the case $n=3$, the domain $\Omega$ is a triangle and it is more convenient to work directly with the barycentric co-ordinate system $\left(b_{0}, b_{1}, b_{2}\right)$ of the point $X \in \Omega$. Thus

$$
X=b_{0} X_{0}+b_{1} X_{1}+b_{2} X_{2}, \quad b_{0}+b_{1}+b_{2}=1 .
$$

In this case the co-ordinte chart representation (3.6) becomes

$$
\phi_{j-1, j}:=\left(u_{j}, v_{j}\right):=\left(\frac{b_{j+1}}{b_{j}+b_{j+1}}, \frac{b_{j-1}}{b_{j}++_{j-1}}\right) .
$$

It should be remarked that the co-ordinate chart is singular at $X=Z_{j}$ and $X=Z_{j-1}$, but for $n \geq 4$ these points are outside the polygonal domain. (In the case $n=4$, the central projections are parallel projections with $Z_{j}$ becoming a point at infinity.) In the case $n=3$, the central projection introduces singularities at the vertices $Z_{j}=X_{j-1}$ and $Z_{\mathrm{j}-1}=X_{\mathrm{j}+1}$. However, these singularities will be removable in the final scheme and will not cause numerical difficulties. Alternatively, a triangular scheme based on a nonsingular parallel projection co-ordinate chart construction can be derived, and this alternative scheme is considered in Section 5.

\section{The polygonal patch}

Let $\Phi_{i-1, i}: \Omega \rightarrow[0,1]^{2}$ be a $C^{k}$ diffeomorphism which maps the vertex $X_{j}$ to $(0,0)$ and the edges $E_{j}(u),-E_{j-1}(1-v)$ to $(u, 0),(0, u)$ respectively. Also, let $\mathrm{P}_{j-1, j}: \Omega \rightarrow I R^{3}$ be defined by the composition

$$
\mathrm{p}_{j-1, j}(X):=\mathrm{q}_{j-1, j}\left(\Phi_{j-1, j}(X)\right), \quad X \in \Omega,
$$

where $\mathrm{q}_{j-1, \mathrm{j}}(u, v),(u, v) \in[0,1]^{2}$, is the $C^{k}$ extension described in Section 2 , see Figure 4. Then, by definition, $\mathrm{p}_{j-1, j}(X), X \in \Omega$, and $\mathrm{q}_{j-1, j}(X), X \in$ $\sigma^{-1}(\delta) \cup \delta$, , see $(2.1)$, form a $C^{\mathrm{k}}$ surface in the sense that there exists a reparameterization in which the surface is $\mathrm{C}^{\mathrm{k}}$. More precisely, $\mathrm{p}_{j-1, j}(X), X \in$ $\Omega$, and $q_{i-1 . i}\left(\Phi_{i-1 . i}(X)\right) \in \Phi_{i-1 . i}^{-1}\left(\sigma^{-1}(\delta) \cup \delta\right)$ form a $\mathrm{C}^{k}$ surface, where $\Phi_{i-1, i}$ defines the reparameterization. Two such surfaces patches, which join with $C^{k}$ continuity under a reparameterization, are said to have a geometric continuous $\mathrm{GC}^{\mathrm{k}}$ join in the CA GD literature. Thus, $\mathrm{p}_{\mathrm{j}-1, \mathrm{j}}$ defines a surface patch on the polygonal domain $\mathrm{ft}$, which, along the edges $E_{j}$ and $E_{j-1}$, has $\mathrm{GC}^{\mathrm{k}}$ joins with the rectangular patch complex. 


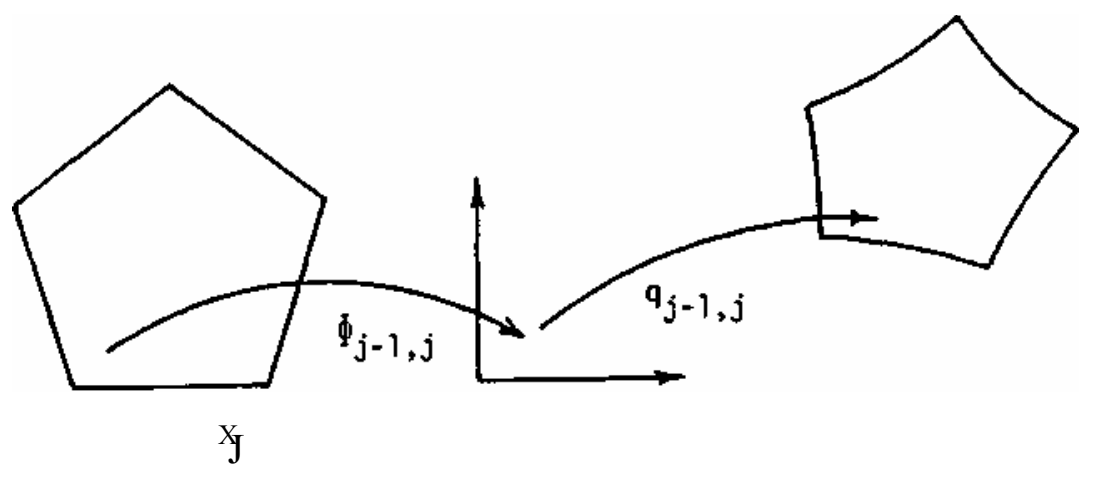

Figure 4: The map $\mathrm{p}_{j-1, j}$

The polygonal patch $\mathrm{p}: \Omega \rightarrow I R^{3}$, is required to have a $G C^{k}$ join with the rectangular patch complex around the entire boundary of $\Omega$. We thus define

$$
\mathrm{p}(X):=\sum_{j=0}^{n-1} w_{j}(X) \mathrm{p}_{j-1, j}(X), X \in \Omega,
$$

where the weights $W_{j}: \Omega \longrightarrow I R$ are $C^{k}$ functions such that

$$
\sum_{j=0}^{n-1} w_{j}(X)=1, w_{j}(X) \geq 0, X \in \Omega,
$$

and, for all $\mathrm{i}=0, \ldots, k$, the derivative maps are such that

$$
\partial^{i} w_{j} \mid E_{\ell}=0, \ell \neq j-1, j ; \ell=0, \ldots, n-1 .
$$

Hence $\mathrm{p}$ is a convex combination of the $\mathrm{P}_{J-1, J}$ with weights $W_{j}$ chosen to be zero to order $k$ on those sides where $\mathrm{P}_{J-1, J}$ does not match the surrounding patch complex.

The unwary reader may feel that the problem is now completely solved, since the work of the previous section suggests that the co-ordinate charts $\phi_{i-1 . i}$ provide appropriate definitions for the diffeomorphisms $\Phi_{i-1 . i}$. However, as observed in [Gregory \& Hahn '87] for the case $k=2$, a convex combination patch of the form (4.2) will not, in general, have a $G C^{k}$ join with the surrounding rectangular patch complex. This problem occurs because of the different diffeomorphisms $\Phi_{i-1 . i}$ defining the reparameterizations of the $G C^{k}$ joins. Thus more care is needed in the construction of the $\Phi_{i-1, i}$.

To investigate this problem further, we consider the join of $\mathrm{q}_{j}$ with $\mathrm{p}$ along the edge $E_{j}$. From (4.1)-(4.4), we can write

$$
\begin{aligned}
\mathrm{p} & =w_{j} \cdot \mathrm{p}_{j-1, j}+w_{j+1} \cdot \mathrm{p}_{j, j+1}+\mathrm{r}_{j} \\
& =w_{j} \cdot\left(\mathrm{q}_{j-1, j} \circ \Phi_{j-l, j}\right)+w_{j+1} \cdot\left(\mathrm{q}_{j, j+1} \circ \Phi_{j-l, j}\right)+\mathrm{r}_{j},
\end{aligned}
$$


where

$$
\partial^{i} r_{j} \mid E_{j}=0, i=0, \ldots, k
$$

and

$$
\left.\begin{array}{l}
\left(w_{j}+w_{j+1}\right) \mid E_{j}=1, \\
\partial^{i}\left(w_{j}+w_{j+1}\right) \mid E_{j}=0, \quad i=1, \ldots, k,
\end{array}\right\} .
$$

Now, by definition, $\mathrm{q}_{j-1, j}$ is a $C^{k}$ extension of $\mathrm{q}_{\mathrm{j}}$ and $\mathrm{q}_{j, j+1}$ is a $C^{k}$ extension of $\mathrm{q}_{j}$ o $\sigma$ across $(\mathrm{s}, 0), 0 \leq \mathrm{s} \leq 1$, see (2.1). Thus $\mathrm{P}_{J-1, J}$ is a $\mathrm{C}^{k}$ extension of $\mathrm{q}_{j} \circ \Phi_{j-1, j}$ and $\mathrm{P}_{J+1, J}$ is a $C^{k}$ extension of $\mathrm{q}_{j} \circ \sigma \circ \Phi_{j, j+1}$ across $-E_{j}(s), 0 \leq \mathrm{s} \leq 1$. Hence $\Phi_{j-1, j}$ and $\sigma 0 \Phi_{j, j+1}$ define the reparameterization functions for the $G C^{k}$ joins. It follows, from (4.5)-(4.8), that if $\Phi_{j-1, j}$ and $\sigma \circ \Phi_{j+1, j}$ agree to order $k$ along $E_{j}$, that is if the $G C^{k}$ joins are identical, then $\mathrm{p}$ has an (identical) $G C^{k}$ join with $\mathrm{q}_{j}$. In fact, the following proposition shows that this condition on the diffeomorphisms can be weakened.

Proposition 1 The polygonal patch $\mathrm{p}$ has a $G C^{k}$ join with $\mathrm{q}_{j}$ if

$$
\partial^{i} \Phi_{j-1, j}\left|E_{j(s)}=\partial^{i}\left(\sigma \circ \Phi_{j, j+1}\right)\right| E_{j(s)}, i=0, \ldots, k-1 \text {, }
$$

that is $\Phi_{j-1, j}$ and $\sigma 0 \Phi_{j, j-1}$ agree to order $k-1$ on $E_{j}(\mathrm{~s}), 0 \leq \mathrm{s} \leq 1$.

Proof. Since $\mathrm{q}_{\mathrm{j}-1, \mathrm{j}}$ is a $\mathrm{C}^{\mathrm{k}}$ extension of $\mathrm{q}_{\mathrm{j}}$ and $\mathrm{q}_{\mathrm{j}, \mathrm{j}+1}$ is a $\mathrm{C}^{\mathrm{k}}$ extension of $\mathrm{q}_{j} 0 \sigma$, then differentiating (4.6) along any direction $U$ using Leibniz' theorem gives

$$
\begin{aligned}
\left.\frac{\partial^{\ell} \mathrm{P}}{\partial U^{\ell}}\right|_{E_{j}}= & \sum_{i=0}^{\ell}\left(\begin{array}{l}
\ell \\
i
\end{array}\right)\left\{\frac{\partial^{\ell-i} w_{j}}{\partial U^{\ell-i}} \frac{\partial^{i} \mathrm{q}_{j} \circ \Phi_{j-1, j}}{\partial U^{i}}\right. \\
& \left.+\frac{\partial^{\ell-i} w_{j+1}}{\partial U^{\ell-i}} \frac{\partial^{i} \mathrm{q}_{j} \circ \sigma \circ \Phi_{j, j+1}}{\partial U^{i}}\right\}\left.\right|_{E_{j}} .
\end{aligned}
$$

Now, from the hypothesis (4.9),

$$
\left.\frac{\partial^{i} \mathrm{q}_{j} \circ \Phi_{j-1, j}}{\partial U^{i}}\right|_{E_{j}}=\left.\frac{\partial^{i} \mathrm{q}_{j} \circ \Phi_{j, j+1}}{\partial U^{i}}\right|_{E_{j}} \quad, i=0, \ldots, k-1 .
$$

Hence, (4.8) and (4.10) give, for $\ell=0, \ldots, k$,

$$
\begin{aligned}
\left.\frac{\partial^{\ell} \mathrm{p}}{\partial U^{\ell}}\right|_{E_{j}} & =\left.\left\{w_{j} \frac{\partial^{\ell} \mathrm{q}_{j} \circ \Phi_{j-1, j}}{\partial U^{\ell}}+w_{j+1} \frac{\partial^{\ell} \mathrm{q}_{j} \circ \sigma \Phi_{j, j-1}}{\partial U^{\ell}}\right\}\right|_{E_{j}}, \\
& =\left.\left\{\frac{\partial^{\ell}}{\partial U^{\ell}} \mathrm{q}_{j} \circ\left[w_{j} \cdot \Phi_{j-1, j}+w_{j+1} \cdot\left(\sigma \circ \Phi_{j, j+1}\right)\right]\right\}\right|_{E_{j}} .
\end{aligned}
$$

This latter result comes after expansion of (4.12) and (4.13), using the chain and product rules, where we again make use of (4.8) and of the fact that $\Phi_{j, j-1}$ and $\left.\sigma \circ \Phi_{j, j+1}\right)$ agree to order $k-1$ on $E_{j}$. For brevity, we omit the details of these expansions. We have thus shown that $\mathrm{p}$ is a $C^{k}$ extension of $\mathrm{q}_{j} \circ\left\lfloor w_{j} . \Phi_{j-1, j}+w_{j+1} \cdot\left(\sigma \circ \Phi_{j, j+1}\right)\right\rfloor$ across $E_{j}$ and hence $\mathrm{p}$ has a $G C^{k}$ join with $\mathrm{q}_{j}-\square$. 


\section{Construction of the diffeomorphism $\Phi_{j-1, j}$}

Proposition 1 shows that $\Phi_{j-1, j}$ must agree with $\sigma \circ \Phi_{j, j+1}$ to order $k-1$ on $E_{J}$ and with $\sigma^{-1} \circ \Phi_{j, j+1}$ to order $k-1$ on $E_{J-1}$.Consider the central projection co-ordinate charts defined in Section 3. Then

$$
\Phi_{j-1, j}=\left(u_{j}, v_{j}\right)
$$

agrees with

$$
\sigma \circ \Phi_{j, j+1}=\left(1-v_{j+1}, u_{j+1}\right)
$$

to order zero on $\mathrm{E}_{J}$, and agrees with

$$
\sigma^{-1} \circ \Phi_{j-2, j-1}=\left(v_{j-1}, 1,-u_{j-1}\right)
$$

to order zero on $E_{J-1}$. Thus $\Phi_{i-1 . i}:=\Phi_{i-1, i}$ is an appropriate definition for the diffeomorphism in the case $k-1$ of Proposition 1, which is the construction used in [Charrot and Gregory'84]. In general, let $\alpha:[0,1] \longrightarrow I R$ be a $C^{k}$ function such that

$$
\alpha^{(i)}(0)=\delta_{i, 0}, \alpha^{(i)}(0)=0, i=0, \ldots k-1 .
$$

Then the following proposition shows that the diffeomorphisim $\Phi_{i-1 . i}$ can be constructed by matching a blend of the charts (4.14)-(4.16).

Proposition 2 Let $\Phi_{i-1 . i}, \mathrm{j}=0, \ldots, \mathrm{n}-1$ be $C^{k}$ diffeomorphisms which respectively match

$$
\psi_{j}(X):=\alpha\left(u_{j}\right)\left(u_{j}, v_{j}\right)+\alpha\left(v_{j+1}\right)\left(1-v_{j+1}, u_{j+1}\right)
$$

to order $k-1$ on $E_{J}$ and

$$
\sigma^{-1} 0 \psi_{j-1}(X):=\alpha\left(u_{j}\right)\left(u_{j}, v_{j}\right)+\alpha\left(v_{j+1}\right)\left(1-v_{j+1}, u_{j+1}\right)
$$

to order $k-1$ on $E j_{-1}$. Then the diffeomorphisms satisfy the $G C^{k}$ conditions (4-9) of Proposition 1.

Proof. At $X=X_{j}, u_{j}=v_{j}=0$ and $v_{j+1}=u_{j-1}=1$. Hence, using (4.17), $\psi_{j}(X)$ and $\sigma^{-1} 0 \psi_{i-1}(X)$ agree to order $k-1$ with $\phi_{i-1 . i}=\left(u_{i}, v_{i}\right)$ at $X_{i}$. Thus the $C^{k}$ functions defined by (4.18) and (4.19) are compatible to order $k-1$ at $X j=E_{j} \cap E_{j-1}$. Hence a $C^{k}$ diffeomorphism $\Phi_{j-1, j}$ can be constructed which matches these $C^{k}$ functions to order $k-1$ on $E_{j}$ and $E_{j-1}$. (An explicit construction involving the central projection co-ordinate charts is given below and an alternative construction involving parallel projection co-ordinate charts is given in Section 5.) Furthermore, since $\Phi_{j-1, j}$ matches $\psi_{j}$ and $\Phi_{j j+1}$ matches $\sigma^{-1}$ o $\psi_{j}$ to order $k-1$ on $E_{j}$, it immediately follows that $\Phi_{j-1, j}$ and $\sigma$ o $\Phi_{j, j+1}$ agree to order $\mathrm{k}-1$ on $E_{j}$. Thus the conditions 
(4.9) of Proposition 1 hold.

Diffeomorphisms $\Phi_{j-1, j}$ must now be constructed which satisfy the conditions (4.18) and (4.19) of Proposition 2. With the central projection coordinate charts, this problem has a simple solution. Observe that

$$
\alpha^{(i)}\left(v_{j+1}\right)\left|E_{j-1}=\alpha^{(i)}\left(u_{j-1}\right)\right| E_{j}=\alpha^{(i)}(1)=0, i=0, \ldots, k-1,
$$

and

$$
\alpha^{(i)}\left(u_{j}\right)\left|E_{j-1}=\alpha^{(i)}\left(v_{j}\right)\right| E_{j}=\alpha^{(i)}(0)=\delta_{i, j,} i=0, \ldots, k-1 .
$$

It then follows that $\Phi_{j-1, j}$ can be denned by the tensor-product like construction

$$
\Phi_{j-1, j}:=\left[\begin{array}{ll}
\alpha\left(u_{j}\right) & \alpha\left(v_{j+1}\right)
\end{array}\right]\left[\begin{array}{cc}
\left(u_{j,} v_{j}\right) & \left(v_{j-1}, 1-u_{j-1}\right) \\
\left(1-v_{j+1}, u_{j+1}\right) & \left(a_{j}, b_{j}\right)
\end{array}\right]\left[\begin{array}{c}
\alpha\left(v_{j}\right) \\
\alpha\left(u_{j-1}\right)
\end{array}\right]
$$

Here, $0 \leq a_{j}, b_{j} \leq 1$ ensures that $\Phi_{j-1, j}: \Omega \rightarrow[0,1]^{2}$. In practice we find that $\left(a_{j}, b_{j}\right)=(0,0)$ produces a satisfactory result and prefer to control the shape of the patch through the choice of the extensions $\mathrm{q}_{j^{-}-j, j}$.

\section{The weight functions $w_{j}$ and $\alpha$}

The polygonal patch construction is summarized as being defined by (4.2), where $\mathrm{p}_{j-1, j}$ is defined by (4.1) and $\Phi_{j-1, j}$ is denned by (4.22). Given the weight functions $w_{j}$ in (4.2) and the weight $\alpha$ in (4.22), then the user has only to supply the $C^{k}$ extensions $\mathrm{q}_{j-1, j}$ of the rectangular patch complex.

We define the weight functions $w_{j}$, which satisfy conditions (4.3) and (4.4), by

$$
w_{j}:=\frac{\prod_{i \neq j-1, j} d_{i}^{k+1}}{\sum_{\ell=0}^{n-1} \prod_{i \neq \ell-1, \ell} d_{i}^{k+1}},
$$

where $d_{i}=d_{j}(X)$ is the perpendicular distance of $X$ from the side $E_{j}$, see Section 3. For $n=3$, these weight functions can be written in terms the barycentic co-ordinates as

$$
w_{j}:=\frac{b_{j}^{k+1}}{\sum_{\ell=0}^{n-1} b_{\ell}^{k+1}} .
$$

(The case $n=3$ also exhibits an alternative polynomial definition for the weights, see Section 5.)

The weight $\alpha$, in the definition of the diffeomorphism $\Phi_{j-1, j}$, must satisfy conditions (4.17). Hermite two point Taylor interpolation then gives

$$
\alpha(s):=(1-s)^{k} \sum_{j=0}^{k-1} \frac{(k-1+j) !}{(k-1) ! j !} s^{j}
$$


as an appropriate definition. Thus, for $k=2$ (curvature continuity),

$$
\alpha(s):=(1-\mathrm{s})^{2}(1+2 \mathrm{~s}) \text {. }
$$

For $k=1$ (tangent plane continuity), we have $\alpha(\mathrm{s})=1-\mathrm{s}$ in the definition (4.22) but, as was observed in the introduction to this section, $\Phi_{i-1 . i}=\phi_{i-1 . i}$ is also valid in this case. Finally, it can be noted that

$$
\alpha(s)+\alpha(1-s)=1
$$

Thus, since $v_{j+1}=1-u_{j}$ for the central projection co-ordinate charts, it follows that (4.18) defines a convex combination of the two charts $\left(u_{i}, v_{j}\right)$ and $\left(1-v_{j+1}, u_{j+1}\right)$.

\section{An alternative triangular patch}

In the triangular domain case $n=3$, the component $\mathrm{p}_{j-1, j}$ of the patch definition (4.2) has singularities at the vertices $X_{j-1}$ and $X_{j+1}$. These are introduced by the singularities of the central projection co-ordinate charts in the definition (4.22) of the diffeomorphism $\Phi_{j-1, j}$. These singularities are removable to order $k$, since the weight $w_{i}$ in (4.2) has a $k+1$ 'st order zero along the edge $E_{j+1}$ which joins $X_{j-1}$ and $X_{j+1}$. Hence the patch definition is numerically stable to order $k$. It is, however, possible to totally avoid the introduction of singularities through the use of parallel projection co-ordinate charts on the triangle. In this case, the construction of $\Phi_{j-1, j}$ as in (4.22) is no longer valid and hence an alternative construction is required.

\section{The parallel projection co-ordinate chart}

For a point $X \in \Omega$, let $u_{j}=u_{j}(X)$ be such that

$$
E_{j}\left(u_{j}\right):=\left(1-u_{j}\right) X_{j}+u_{j} X_{j+1}
$$

is the point of intersection of the edge $E_{j}$ with the ray through $X$ parallel to the side $E_{j-1}$. Also, let $v_{j}=v_{j}(X)$ be such that

$$
E_{j-1}\left(1-v_{j}\right):=\left(1-v_{j}\right) X_{j}+v_{j} X_{j-1}
$$

is the point of intersection of the edge $E_{j-1}$ with the ray through $X$ parallel to the side $E_{j}$. Then

$$
\phi_{j-1, j}(X):=\left(u_{j}(X), v_{j}(X)\right)
$$

defines the co-ordinate chart, see Figure 5. As in the case of the central projection, the co-ordinate chart maps $X_{j}$ to $(0,0)$ and the two edges $E_{j}(\mathrm{u})$ 


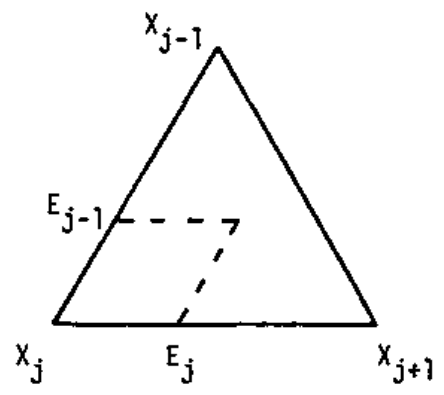

Figure 5: Parallel projection co-ordinate chart construction

and $E_{j-1}(1-\mathrm{v})$ onto $(\mathrm{u}, 0)$ and $(0, \mathrm{v})$ respectively. Also, for $\mathrm{n}=3$, the chart maps the triangle $\Omega$ into $[0,1]^{2}$. However, for $n \geq 5$ there are points $X \in \Omega$ for which $\Phi_{j-1, j}(X) \notin[0,1]^{2}$. Hence we do not propose the use of the parallel projection in the case $n \geq 5$. For $n=3$ the co-ordinate chart is conveniently computed in terms of the barycentric co-ordinate system as

$$
\phi_{j-1, j}:=\left(u_{j,} v_{j}\right):=\left(b_{j+1}, b_{j-1}\right)
$$

\section{The diffeomorphism $\Phi_{j-1, j}$}

Conditions (4.20), which hold for the central projection co-ordinate charts, are not valid for the parallel projection co-ordinate charts. Hence the diffeomorphism $\Phi_{j-1, j}$ cannot be constructed as in (4.22). Now

$$
\left(u_{j+1,} v_{j+1}\right)=\left(v_{j,} 1-u_{j}-v_{j}\right),\left(u_{j-1}, v_{j-1}\right)=\left(1-u_{j}-v_{j}, u_{j}\right)
$$

for the parallel projections on the triangle. Hence, in terms of the variables $\left(u_{j}, v_{j}\right)$, Proposition 2 requires the construction of $\Phi_{j-1, j}$ which matches

$$
\psi_{j}=\alpha\left(u_{j}\right)\left(u_{j,} v_{j}\right)+\alpha\left(1-u_{j}-v_{j}\right)\left(u_{j,}+v_{j,} v_{j}\right)
$$

to order $\mathrm{k}-1$ on $v_{j}=0$, and

$$
\sigma^{-1} o \psi_{j-1}=\alpha\left(v_{j}\right)\left(u_{j}, v_{j}\right)+\alpha\left(1-u_{j}-v_{j}\right)\left(u_{j} u_{j}+v_{j}\right),
$$

to order $k-1$ on $u_{j}=0$. Now for $0 \leq \ell, m \leq k-1$,

$$
\left.\partial_{l, m} \psi_{j}\right|_{(00)}=\left.\partial_{\ell, m}\left(\sigma^{-1} o \psi_{j-1}\right)\right|_{(0,0)}=\left.\partial_{\ell, m} \phi_{j-1, j}\right|_{(0,0)}=\left.\partial_{\ell, m}\left(u_{j,} v_{j}\right)\right|_{(\cap n)} .
$$

which shows that $\psi_{i}$ and $\sigma^{-1} o \psi_{i-1}$ are compatible to order $k-1$ in each of the variables $u_{i}$ and $v_{i}$ at $\left(u_{i}, v_{i}\right)=(0,0)$. We can thus define $\Phi_{i-1 . i}$ by the 
Boolean sum Taylor interpolant

$$
\begin{aligned}
\Phi_{j-1, j}:= & \sum_{\ell=0}^{k-1} \frac{u_{j}^{\ell}}{\ell !} \partial_{\ell, o}\left(\sigma o \psi_{j-1}\right)\left|\left(0, v_{j}\right)+\sum_{m=0}^{k-1} \frac{v_{j}^{m}}{m !} \partial_{0, m} \psi_{j}\right|\left(u_{j,} 0\right) \\
& -\left.\sum_{\ell=0}^{k-1} \sum_{m=0}^{k-1} \frac{u_{j}^{\ell}}{\ell !} \frac{v_{j}^{m}}{m !} \partial_{\ell, m} \phi_{j-1, j}\right|_{(0,0)}
\end{aligned}
$$

In particular, with $\alpha$ defined by (4.25), then it can be shown that for $k=1$ (tangent plane continuity)

$$
\Phi_{j-1, j}:=\phi_{j-1, j}:=\left(u_{j,} v_{j}\right),
$$

and for $k=2$ (curvature continuity)

$$
\Phi_{j-1, j}:=\left(u_{j}+9 u_{j}^{2} v_{j}-8 u_{j}^{3} v_{j}, v_{j}+9 u_{j} v_{j}^{2}-8 u_{j} v_{j}^{3}\right) .
$$

The triangular patch is constructed as in (4.2), where the diffeomorphism $\Phi_{j-1, j}$ in (4.1) is defined by the above Boolean sum construction. The weights $w_{j}$ in (4.2) can be computed by the rational form (4.24). Alternatively, the polynomial weight

$$
w_{j}:=b_{j}^{k-1} \sum_{l=0}^{k} \sum_{m=0}^{k} \frac{(k+l+m) !}{k ! l ! m !} b_{j-1}^{l} b_{j+1}^{m}
$$

can be used, since it satisfies properties (4.3) and (4.4) on the triangular domain $\Omega$. In this case the triangular patch $\mathrm{p}$ will be a polynomial form in the barycentric co-ordinates, if the $\mathrm{q}_{j-1, j}$ extensions of the rectangular patch complex are polynomial.

\section{Numerical Implementation}

We consider the implementation of the polygonal patch schemes for the specific case of uniform bicubic B-spline surfaces which contain polygonal holes. Two possible arrangements for the control points of $C^{2}$ bicubic B-spline patch complexes $\mathrm{q}_{j}, j=0, \ldots, n-1$, about triangular holes are shown in figures 6 and 7. The first example gives one patch adjacent to each edge of a traingular hole and involves the use of a central control point of multiplicity four. The second example gives two patches adjacent to each edge. Clearly, any number of patches adjacent to the hole can be obtained by the addition of further control points. Also, $n$-sided holes can be obtained by generalizations of these arrangements of the control points.

Given that the surrounding patch complex has a $C^{2}$ bicubic B-spline representation, then it is natural to construct the rectangular patch extension $\mathrm{q}_{j-1, j}$ as a bicubic B-spline surface. In the first example, the extension will be 


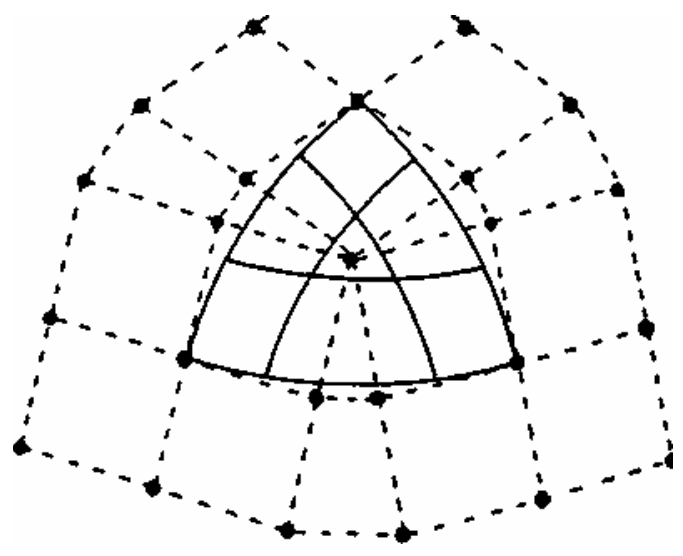

Figure 6: Six bicubic B-spline patches about a triangular hole

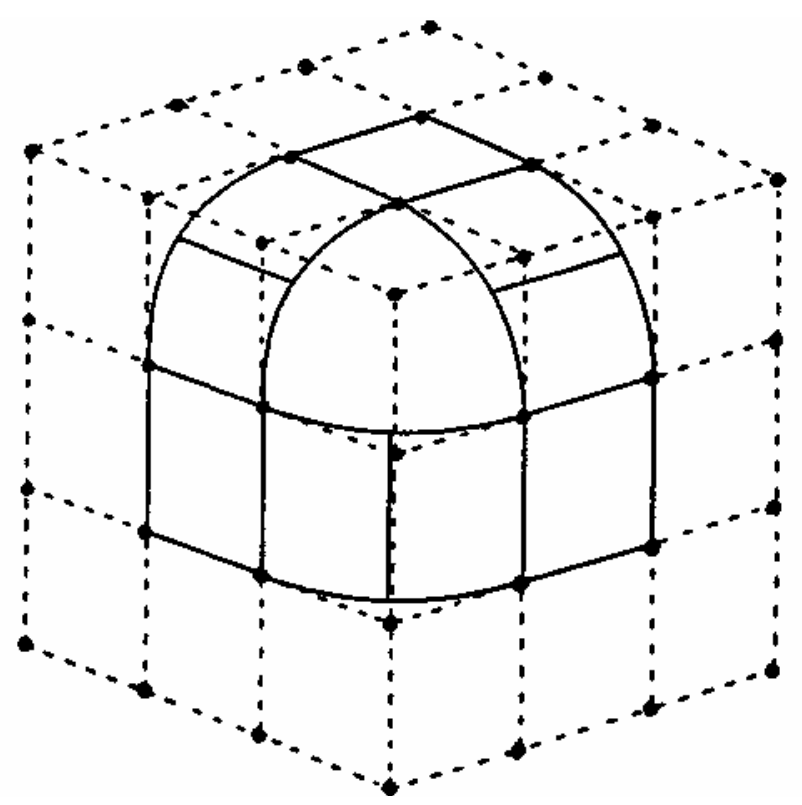

Figure 7: Nine bicubic B-spline patches about a triangular hole 
a single patch, requiring the definition of an additional control point. In the second example, the extension will consist of four bicubic B-spline patches, requiring the definition of four additional control points. Additional control points will be required for each extension $\mathrm{q}_{j-1, j}, j=0, \ldots, n-1$, and can be considered as degrees of freedom which allow some control over the shape of the final polygonal patch. Also, the diffeomorphism $\Phi_{j-1, j}$, which is used in the reparameterization of $\mathrm{q}_{j-1, j}$, involves a degree of freedom $\left(a_{j}, b_{j}\right)$ to manipulate the shape of the polygonal surface. However, experimental results indicate that this degree of freedom may introduce undesirable "hump" effects around the central region of the surface. Hence, in practice, we set $\left(a_{j}, b_{j}\right)=(0,0), j=0, \ldots, n-1$.

In order to plot the polygonal patch given by (4.1) and (4.2), it is convenient to reparameterize it as a complex of rectangular patch mappings. This is achieved by quadrilateral subdivision of the polygonal domain about its centre, introducing additional bilinear maps from the unit square onto the quadrilaterals. The problem of calculating normals and curvatures of the patch on the polygonal domain is solved here by adopting a "procedural"method. Alternatively, a numerical differentiation technique applied to the rectangular patch mappings could be used and is probably the simplest technique to apply in practice.

We conclude by giving some model examples, where the control points are arranged to give two bicubic B-spline patches adjacent to each edge. Figure 8 shows the filling of a triangular hole, displayed using a standard shading technique based on the use of unit normals. Figure 9(a) shows the same surface plotted with a Gaussian curvature shading map. Figure 9(b) shows a $C^{\mathrm{l}}$ fill of the triangular hole as a comparison. The curvature shading technique is also used for the lighting model calculation so that the lack of curvature continuity across the boundary of the triangular patch is clearly displayed. Figures 10 and 11 show a similar display for the case of a pentagonal patch. The final figure 12 shows the combination of a triangular and pentagonal patch within a $C^{2}$ rectangular patch complex.

\section{Acknowledgements}

This work was supported by the ACME Directorate of the Science and Engineering Research Council, with the grant GR/E 25092. We are also pleased to acknowledge the help of P. K. Yuen, who wrote the software for the curvature lighting model.

\section{References}

[1] Charrot, P. and Gregory J. A. (1984), A pentagonal surface patch for 
computer aided geometric design, Computer Aided Geometric Design 1, 87-94.

[2] Gregory, J. A. and Hahn, J. M. (1987a), Polygonal patches of high order continuity, Technical Report TR/01/87, Mathematics \& Statistics Department, Brunei University.

[3] Gregory, J. A. and Hahn, J. M. (1987b), Geometric continuity and convex combination patches, Computer Aided Geometric Design 4, 79-89.

[4] Gregory, J. A. and Hahn, J. M. (1989), A $C^{2}$ polygonal surface patch, Computer Aided Geometric Design 6, 69-75.

[5] Gregory, J. A. Lau, V. K. H. and Zhou, J. (1990), Smooth parametric surfaces and n-sided patches, in: W. Dahmen, M. Gasca and C. A. Micchelli, eds., Computation of Curves and Surfaces, NATO ASI Series C, Vol. 307, Kluwer Academic Publishers.

[6] Hahn, J. M. (1989), Geometric continuous patch complexes, Computer Aided Geometric Design 6, 55-67.

[7] Varady, T. (1991), Overlap patches: a new scheme for interpolating curve networks with $n$-sided regions, Computer Aided Geometric Design 8, 727. 


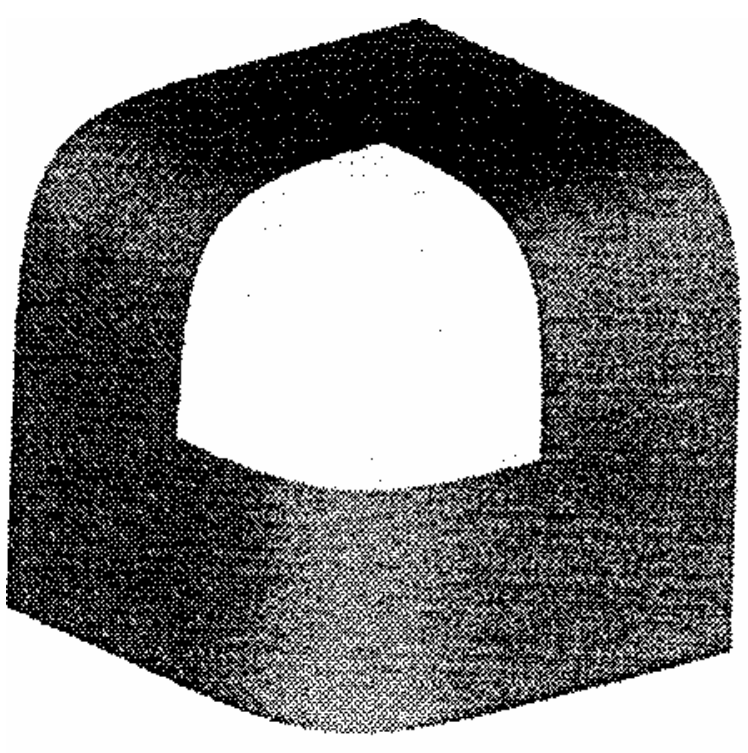

Figure 8(a)

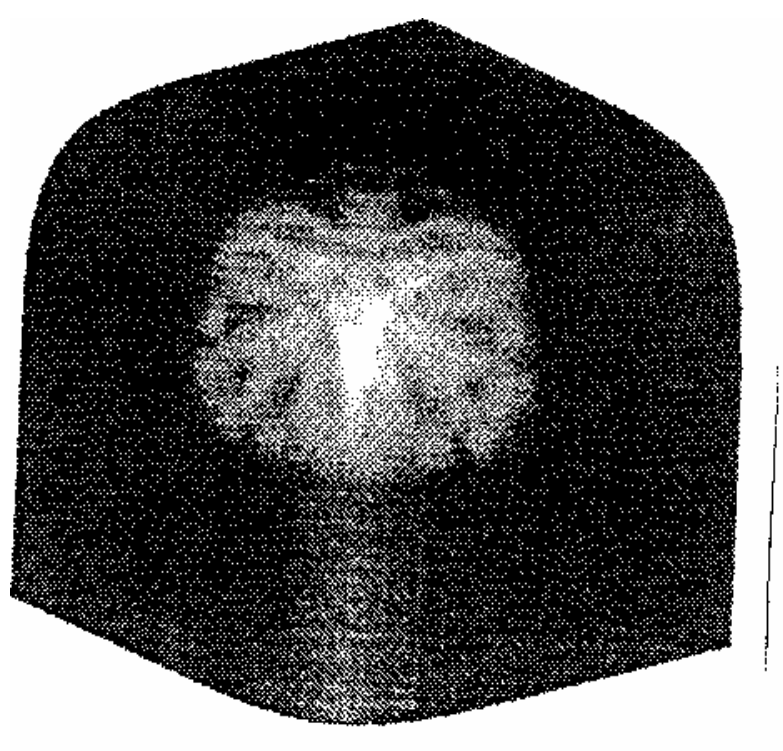

Figure 9(a)

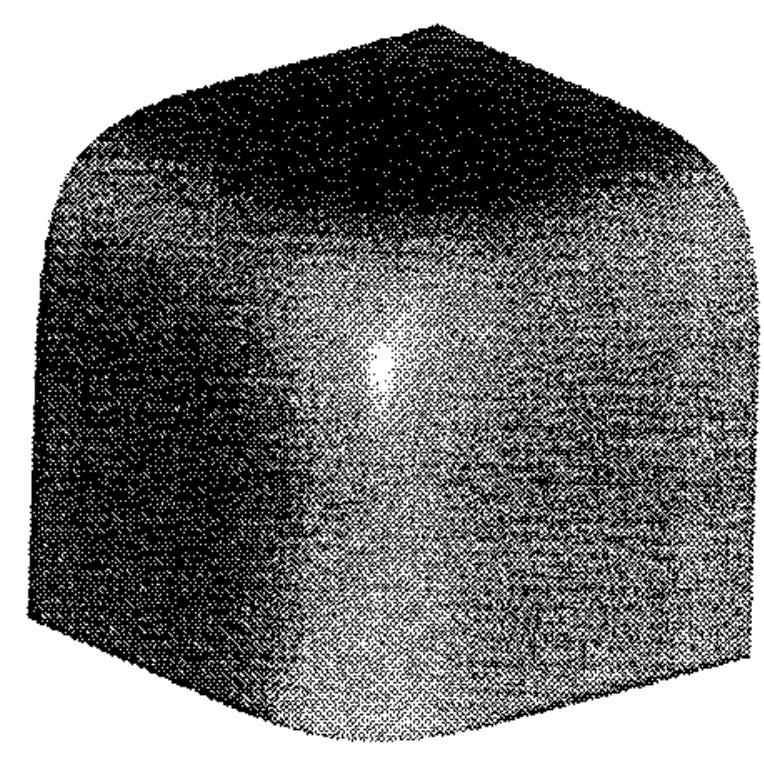

Figure 8(b)

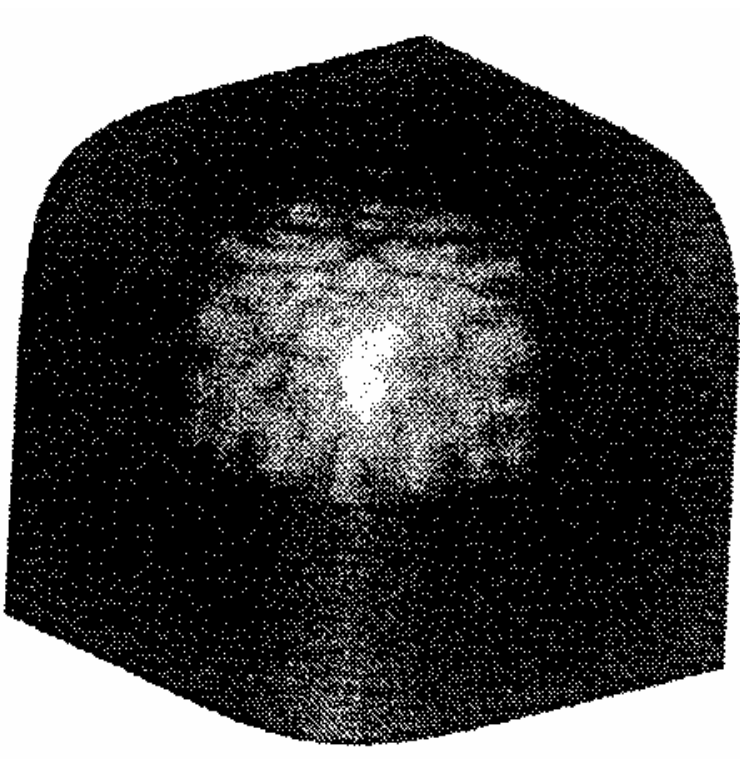

Figure9(b) 


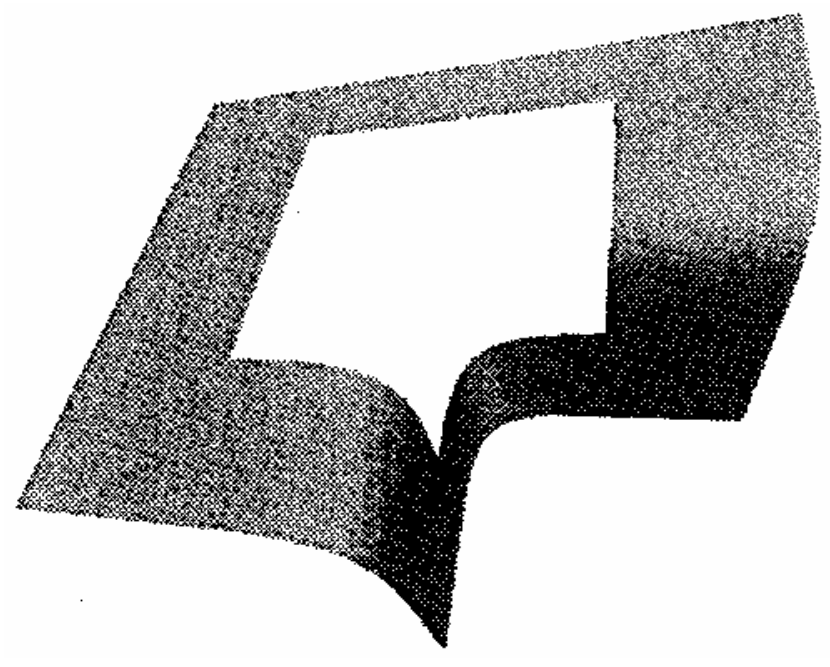

Figure 10(a)

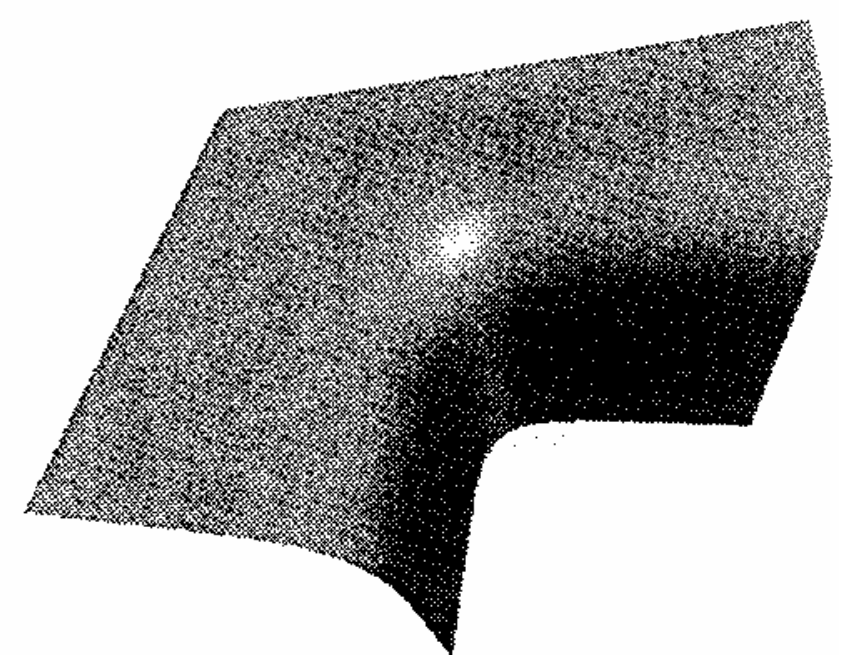

Figure 10(b)

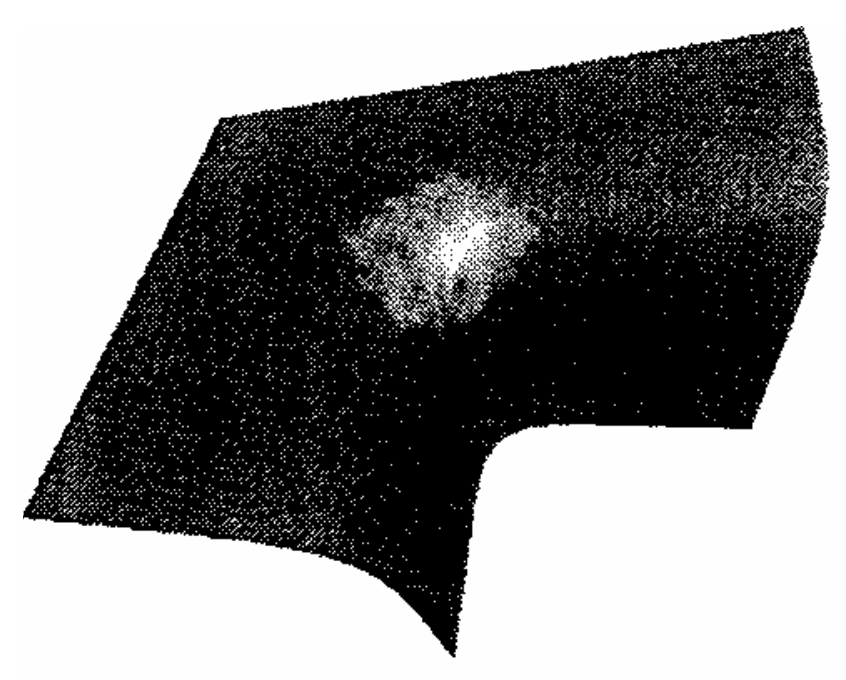

Figure 11 (a)

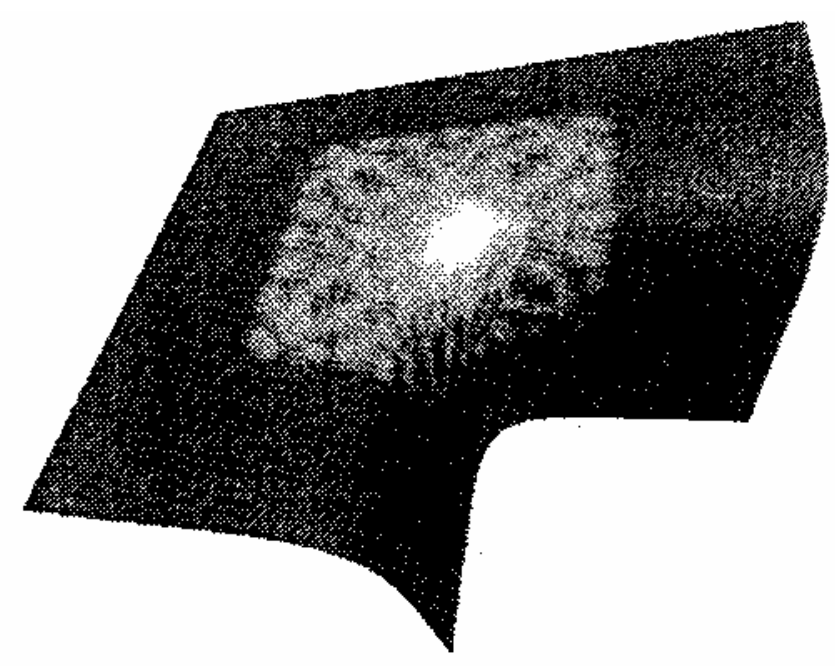

Figure 11(b) 


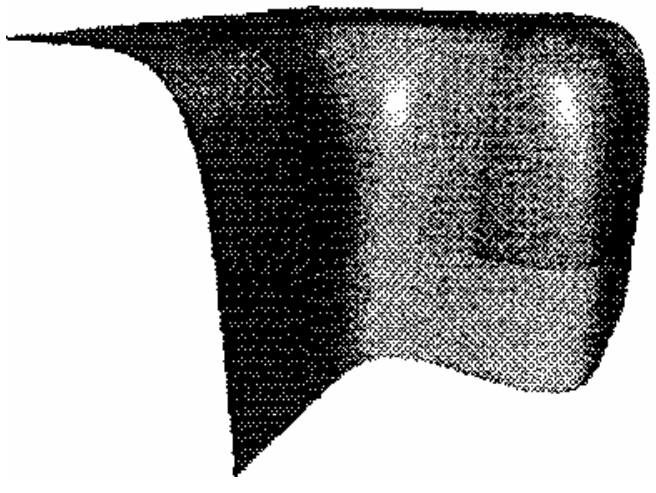

Figure 12(a)

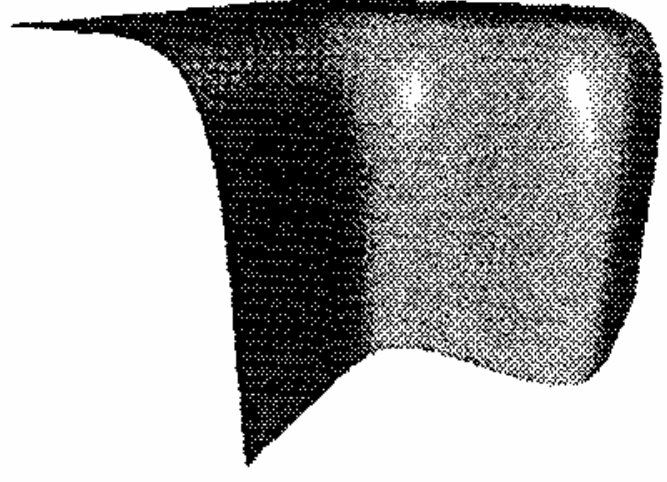

Figure 12(b) 


\section{NOT TO BE REMOVED}

FROM THE LIBRARY

XB 23214341

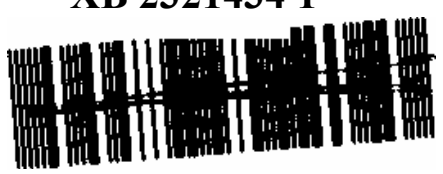

